THURSDAY, DECEMBER I8, I913.

\section{PRINCIPLES OF MATHEMATICS.}

Principia Mathematica. By Dr. A. N. Whitehead, F.R.S., and Bertrand Russell, F.R.S. Vol. iii. Pp. $\mathrm{x}+49$ I. (Cambridge: University Press, I9I3.) Price $21 s$, net.

THE third volume of this work has followed very closely upon the second, which was only published last year, and is in every respect a worthy successor to it. It is mainly concerned with the theory of series, which was begun in the second volume, and then proceeds to the theory of measurement. A further and final volume will deal with geometry. To some extent the treatment has been influenced by the coming volume, especially in the section devoted to the theory of measurement. For the same reason, a special section is included, containing the theory of cyclic families, such as the angles about a given point in a given plane.

That the monumental task which the authors have undertaken should already have reached this stage is almost incredible. For they are in effect creating a new science, with a symbolism of its own, quite foreign to mathematics, which develops naturally as the work proceeds. It is scarcely necessary to point out that the Principia does not concern itself with the development of mathematics, as understood by the mathematician, but solely with the logical deduction of the propositions of mathematics from merely logical foundations. It represents, in one aspect, the culmination of the movement, which has swept over mathematics of late years, towards a rigorous examination of its fundamental premises. To such a work there is always the disadvantage inherent in a new symbolism, but a symbolism is essential to its development, and the authors employ the method which inflicts the minimum of labour on the reader: no symbol or abbreviation is employed until it becomes essential, and then its very recurrence fixes it in the mind of the reader.

The general scope of the volume has been indicated already, and it only remains to consider the detailed treatment adopted. Well-ordered series are considered first, as possessing many important properties not shared by series in general. In particular, they obey a process of transfinite induction, which is an extended form of mathematical induction, differing, however, in the fact that it deals with the successors of classes instead of single terms. On the whole, Cantor's treatment is followed closely, but an exception is made in dealing with Zermolo's theorem, and in the NO. 23O3, VOL. 92] cases where Cantor assumes the multiplicative axiom. The writers emphasise the dubious character of much of the ordinary theory of transfinite ordinals, depending on the fact that it is founded on a proposition requiring this axiom. Ordinal numbers are defined as the relationnumbers of well-ordered series, after Cantor, serial numbers being the relation-numbers of series in general. Products of an ordinal number of ordinal numbers are not in general ordinal numbers, although the sums are. The treatment of sums and products contains much new matter. Perhaps the most interesting part of the work is the authors' solution of the paradox proposed by Burali-Forti in 1897 , relating to the greatest ordinai number. It appears that in any one type there is no greatest ordinal number, and that all the ordinal numbers of a given type are exceeded by those of higher types.

An important section is concerned with the distinction of finite and infinite as applied to series and ordinals. The distinguishing properties of finite ordinals are then established. It does not appear that a proof can be found of the existence of alephs or $\omega$ 's with infinite suffixes. For the type increases with each successive existencetheorem, and infinite types appear to have no meaning. The treatment of the theory of ratio and measurement is quite new. The quantities are regarded as "vectors" in a generalised sense, so that ratios can hold between relations. The hypothesis that the vectors concerned in any context form a group is not prominent. The theory of measurement is a combination of two other theories, one a pure arithmetic of ratios and real numbers, and the other a pure theory of vectors. If the axiom of infinity is assumed, great difficulties in connection with the existence-theorems are avoided. But the authors have endeavoured to get rid of the assumption, for, as they point out, it does not seem proper to make the theory of a simple ratio like $2 / 3$ depend on the fact that the universe contains an infinite number of objects.

The theory of ratio and measurement is actually the most important part of the volume, but it is impossible in a brief review to do justice to it. Yet it must be said that the publication of this volume is a landmark in the theory, and the authors have earned the sincere thanks of all mathematicians who are interested in the logical foundations of their subject. The printing must have been a peculiarly difficult task, on account of the nature of the symbols, and the Cambridge University Press is to be congratulated on the manner in which this work, like its predecessor, has been produced. 